\title{
AdsorÇão de Metais Pesados em SERRAGem de MAdeira TRATADA COM ÁCIDO CÍTRICO
}

\section{HEAVY METALS SORPTION ON TREATED WOOD SAWDUST}

\author{
RAFAEL FALCO RODRIGUES \\ Graduando do Curso de Engenharia Metalúrgica e bolsista do Núcleo de Valorização em Materiais Minerais da UFOP

\section{RAFAEL LOPES TREVENZOLI} \\ Graduando do Curso de Engenharia Metalúrgica e bolsista do Núcleo de Valorização em Materiais Minerais da UFOP
}

\section{LUCIANO RodRIGo GoMEs SANTOS}

Químico Industrial (UFOP). Mestrando em Engenharia de Materiais (REDEMAT/UFOP) e bolsista do Núcleo de

Valorização em Materiais Minerais da UFOP

\section{VERSIANE ALBIS LEÃO}

Engenheiro Metalúrgico (UFOP). Mestre e Doutor em Engenharia Metalúrgica e de Minas (UF MG). Professor do Departamento de Engenharia Metalúrgica e de Materiais da UFOP. Pesquisador do Núcleo de Valorização em Materiais Minerais da UFOP

\section{VAGNER ROBERTO BOTARO}

Bacharel, Mestre e Doutor em Química (USP). Pós-Doutor em Química. (Université Grenoble, França). Professor do Departamento de Química da UFOP

Recebido: 15/03/05 03/08/05

\section{RESUMO}

Neste estudo, avaliou-se a capacidade da madeira Paraju (Manilkara longifolia), modificada quimicamente, para a retenção de íons metálicos. A modificação refere-se a um tratamento com ácido cítrico que leva à reação de grupos carboxilato com a celulose da biomassa. A introdução desses grupos foi observada através de espectrometria de infravermelho. Foram realizados ensaios em sistemas de batelada contendo cádmio e cobre. Os ensaios foram conduzidos em condiçōes de equilíbrio, ajustados segundo o modelo de Langmuir. Obteve-se um carregamento máximo nos sistemas contendo apenas um metal, de 0,56 mmol-Cd/g e 0,94 mmol-Cu/g. O carregamento de cádmio reduziu-se de $0,56 \mathrm{mmol}-\mathrm{Cd} / \mathrm{g}$ para $0,21 \mathrm{mmol}-\mathrm{Cd} / \mathrm{g}$, à medida que a concentração de cobre cresceu no sistema mostrando que existe uma competição entre os metais pelos grupos de troca.

PALAVRAS-CHAVE: Metais, biosorventes, efluentes.

\section{ABSTRACT}

In the present work, the exchange capacity of Manilkara longifolia sawdust, chemically modified, was studied. The modification was carried out by a chemical treatment with citric acid, which introduced carboxilate groups in the material surface. The introduction of these groups was confirmed by infrared spectroscopy. Cadmium and copper sorption was studied batchwise. Isotherms were drawn and adjusted to the Langmuir model. Maximum loadings of $0.56 \mathrm{mmol} \mathrm{Cd} / \mathrm{g}$ and $0.94 \mathrm{mmolCu} / \mathrm{g}$ were achieved in single metal adsorption. Cadmium uptake was reduced from $0.56 \mathrm{mmolCd} / \mathrm{g}$ to $0.21 \mathrm{mmolCd} / \mathrm{g}$ as the copper concentration increased in the reaction system. These results show that there is a competition between the two metals for the exchange sites.

KEYWORDS: Heavy metals, biosorbents, effluents.

\section{INTRODUÇÃO}

A industrialização de muitas regiōes aumentou a geração de efluentes contendo metais pesados, como o cádmio, que é um dos mais tóxicos. A maioria dos metais pesados é prejudicial a uma variedade de espécies vivas, incluindo os seres humanos. Mesmo em baixos teores, o metal causa sérias doenças, principalmente nos rins e nos ossos. A contaminação ocorre através de inalação ou ingestão, esta última causada, principalmente, pela capacidade das plantas em bioacumu lar o metal em altas proporçôes (Marques et al, 1999; Yalçinkaya et al, 2002). Em virtude disso, a resolução CONAMA 357/2005, estabelece que a concentração máxima de cádmio em águas das classes 1 e 2 é 0,001 mg/L sendo que o teor máximo emitido por qualquer efluente industrial, desde que não haja alteração de classe na água que o recebe, não deve ser maior que $0,2 \mathrm{mg} / \mathrm{L}$.

Historicamente, os casos sérios de contaminação por cádmio envolveram a indústria metalúrgica. $\mathrm{O}$ caso mais grave ocorreu na bacia do rio Jintsu, no Japão, onde se cultivava arroz irrigado com água contaminada, proveniente de uma mineração e metalurgia de zinco a montante da área de cultivo. Centenas de pessoas, particularmente as mais idosas, con- 
traíram uma doença degenerativa dos ossos conhecida como itai-itai. Aparentemente alguns íons $\mathrm{Ca}^{+2}$ foram substituídos por íons $\mathrm{Cd}^{+2}$ uma vez que seus raios ônicos são muito próximos. Os ossos tornaram-se porosos e fraturavam-se com facilidade. Por outro lado, exposiçôes crônicas ao metal resultam em doenças nos rins (Baird, 1999).

Métodos convencionais de tratamento de efluentes contendo metais pesados como precipitação, oxidação ou redução, filtração, troca iônica, tratamento eletroquímico, dentre outros, são muitas vezes restritos por inviabilidade técnica e/ou econômica, especialmente quando os metais estão dissolvidos em grandes volumes de água e em concentraçōes relativamente baixas. Por exemplo, processos de precipitação nem sempre garantem que a concentração metálica esteja dentro dos níveis máximos estabelecidos pela legislação específica, além de produzir, em alguns casos, resíduos difíceis de serem tratados. Por outro lado, resinas poliméricas (de troca-iônica ou quelantes) são bastante eficientes, porém de alto custo (Benguella and Benaissa, 2002; Yalçinkaya et al, 2002).

Diferentes tipos de biomassa, como produtos agrícolas e madeiras, têm a capacidade de reter íons metálicos através de adsorção, levando vantagem sobre resinas comerciais, por serem viáveis economicamente, biodegradáveis e provirem de recursos renováveis (Vaughan et al, 2001). Esta capacidade pode ser aumentada significativamente quando a biomassa é modificada quimicamente. Marshall et al (1999) demonstraram isto quando submeteram casca de soja a uma extração alcalina $(\mathrm{NaOH})$, seguida de uma modificação com ácido cítrico em temperatura elevada e observaram o aumento da capacidade da biomassa em reter cádmio e cobre. Vaughan et al (2001) aplicaram os mesmos tratamentos químicos em sabugo de milho e demonstraram que, dependendo do metal $(\mathrm{Cd}, \mathrm{Cu}, \mathrm{Zn}$ e Ni) a ser adsorvido e do tratamento utilizado, a capacidade de carregamento do material é equivalente ou melhor do que o carregamento de determinadas resinas comerciais. Gaballah et al (1994) fizeram um estudo de adsorção de cádmio em casca de pinho, observando que, para ensaios com concentração inicial de até 100 ppm de cádmio, a capacidade de adsorção é máxima para valores de $\mathrm{pH}$ iguais ou superiores a 5 .

Devido a estas considerações, a procura por novos adsorventes que sejam viá- veis do ponto de vista técnico/econômico está focada nos biomateriais, como fungos, bactérias, algas, madeiras, subprodutos agrícolas e outros. O termo geral "biossorção" tem sido empregado para descrever a propriedade desses materiais em reter íons de soluções aquosas, sendo por isso considerados promissores na remoção de metais pesados de efluentes industriais (Pagnanelli et al, 2001).

A biossorção resulta de interações eletrostáticas e também da formação de complexos entre os íons metálicos e os grupos funcionais presentes na superfície celular, quando estes exibem alguma afinidade química pelo metal. A identificação dos grupos funcionais é importante para determinar os mecanismos responsáveis pela ligação dos metais na estrutura destes materiais. A espectrometria na região do infravermelho é uma das técnicas empregadas para determinar os mecanismos responsáveis por estas interaçōes (Kappor and Viraraghavan, 1997). Kapoor e Viraraghavan (1997) submeteram o fungo Aspergillum Niger a diferentes tratamentos químicos para modificar os grupos funcionais responsáveis pela retenção de íons metálicos, os quais foram caracterizados por espectrometria de infravermelho. Grupos funcionais como carboxilato, fosfato e amino foram considerados como responsáveis pelo processo de biossorção.

Neste trabalho, foi realizada uma modificação química com ácido cítrico em serragens da madeira Paraju (Manilkara longifólia), com o objetivo de introduzir grupos carboxilato em sua estrutura, os quais são responsáveis por um aumento significativo da capacidade da madeira em adsorver metais. Esses grupos funcionais foram caracterizados através de espectrometria na região do infravermelho, que indicou um aumento da concentração de grupos - $\mathrm{COOH}$ na estrutura do material. Foram realizados ensaios de equilíbrio, em sistemas contendo cádmio ou cobre e em sistemas contendo os dois metais, sendo que os primeiros foram ajustados segundo o modelo de Langmuir.

\section{DESENVOLVIMENTO EXPERIMENTAL}

\section{Preparação da madeira}

Foram coletadas amostras de serragem de Paraju (Manilkara longifólia) na região de Ouro Preto (MG). O material foi peneirado a fim de se obter uma granulometria mais homogênea, sendo selecionada a faixa de $0,074 \mathrm{~mm}$ para a condução dos ensaios de adsorção.

Um grama de serragem foi reagida com $20 \mathrm{~mL}$ de uma solução $0,1 \mathrm{~mol} / \mathrm{L}$ $\mathrm{NaOH}$. A mistura foi agitada por $2 \mathrm{~h}$ e o líquido foi descartado. A madeira foi então lavada, com água deionizada, repetidas vezes, e seca à $55^{\circ} \mathrm{C}$, durante $24 \mathrm{~h}$. Esse procedimento permitiu retirar pigmentos da madeira, que poderiam afetar a qualidade do efluente, já que o líquido extraído possuía a cor marrom. À madeira lavada com $\mathrm{NaOH}$ foi adicionada a uma solução 1,2 mol/L de ácido cítrico na proporção de $8,3 \mathrm{~mL}$ de solução por grama de madeira. A mistura foi agitada durante 30 min e o líquido foi descartado. A madeira foi seca a uma temperatura de $55^{\circ} \mathrm{C}$. Após $24 \mathrm{~h}$, a temperatura foi aumentada para $120^{\circ} \mathrm{C}$ e mantida durante 90 min. A madeira foi retirada, lavada com água deionizada $\left(60-80{ }^{\circ} \mathrm{C}\right)$, repetidas vezes, e seca a $55^{\circ} \mathrm{C}$, durante $24 \mathrm{~h}$.

\section{Experimentos de equilíbrio}

A biossorção de íons cádmio e cobre foi estudada em condiçôes de equilíbrio. Para os ensaios contendo apenas um metal: $50 \mathrm{~mL}$ de solução com diferentes concentraçōes (entre 100 e $1000 \mathrm{mg} / \mathrm{L}$ ) foram agitadas com $0,5 \mathrm{~g}$ de madeira em agitador orbital. As misturas foram agitadas durante 24 h e o $\mathrm{pH}$ mantido constante em 5 com adição de $\mathrm{NaOH}$ ou $\mathrm{HCl}$. Em seguida, as misturas foram filtradas e as soluçôes analisadas em espectrofotômetro de absorção atômica Perkin Elmer modelo Analyst 100, para determinar as concentraçōes iniciais e finais dos metais em solução. $\mathrm{O}$ mesmo procedimento foi realizado para sistemas contendo os dois metais, onde, às soluções de cádmio foram adicionados $200 \mathrm{ou}$ $600 \mathrm{mg} / \mathrm{L}$ de cobre.

Com os dados obtidos, foi calculado o carregamento de cada amostra, ou seja, a quantidade do metal (mmol) adsorvida por grama de madeira, através da expressão:

$\mathrm{q}_{\mathrm{eq}}=\frac{\left(\mathrm{C}_{0}-\mathrm{C}_{\mathrm{eq}}\right) * \mathrm{~V}}{\mathrm{M}}$

Onde $\mathrm{q}_{\mathrm{eq}}$ é o carregamento, $\mathrm{C}_{0}$ e $\mathrm{C}_{\mathrm{eq}}$ as concentrações iniciais e finais do metal em solução $(\mathrm{mol} / \mathrm{L})$, respectivamente. $\mathrm{V}$ é o volume da solução e $\mathrm{M}$ a massa da madeira.

Determinadas as concentrações do metal e o carregamento, foram traçadas 
isotermas de adsorção, onde cada curva é construída com o carregamento da madeira em função da concentração de cádmio final, em solução.

\section{RESULTADOS E DISCUSSÃO}

\section{Caracterização do material}

O fenômeno de biossorção ocorre devido à presença de diversos grupos funcionais que constituem a biomassa tais como celulose, polioses, proteínas e lignina. Neste trabalho, não foi conduzida uma análise química para confirmar a presença dos grupos carboxilato na estrutura da serragem. Para confirmar a reação da biomassa com o ácido cítrico, foi conduzida a modificação de uma amostra de celulose, seguindo o mesmo procedimento conduzido para a amostra de Paraju. A celulose foi submetida à análise de espectroscopia na região do infravermelho, para comprovar a presença destes grupos na celulose modificada. O espectro é apresentado na Figura 1.

A espectrometria na região do infravermelho é uma técnica normalmente utilizada para identificar a presença de grupos funcionais. Os ácidos carboxíli$\cos (-\mathrm{COOH})$ possuem o grupo carbonila $(\mathrm{C}=\mathrm{O})$ que apresenta banda de absorção característica na região entre 1100 e $1800 \mathrm{~cm}^{-1}$. Mais especificamente, a carbonila pertencente a um grupo $\mathrm{COOH}$, não ionizado, apresenta banda de absorção na região de 1700 a $1750 \mathrm{~cm}^{-1}$, de acordo com os estudos de Kappor \& Viraraghavan (1997) e Drake et al (1996). Como mostra a Figura 1, grupos carbonila não são observados na celulose sem tratamento $\left(1733 \mathrm{~cm}^{-1}\right)$. Após a reação com o ácido cítrico, essa banda aparece no espectro da celulose modificada, indicando a incorporação do ácido cítrico, ou de seu éster. Marshal et al (1999) citam que o tratamento com ácido cítrico seguido do aquecimento do sistema leva à formação de produtos de condensação tal como o anidrido de ácido cítrico. $\mathrm{O}$ anidrido reage com os grupos hidroxila da matriz celulósica e introduz grupos carboxilato (que não reagiram com a celulose) no material (Figura 2) o que permite a adsorção de cátions. A esta última reação é atribuída a presença dos grupos de troca na madeira modificada. Como o espectro de infravermelho indica a região de vibração dos grupos carbonila, comuns tanto a ésteres quanto a ácidos carboxílicos, a determinação do



Figura I - Espectros de infravermelho por transformada de Fourier das amostras de celulose, celulose modificada e ácido cítrico número (não a concentração) de grupos carboxilato, capazes de adsorver os metais, via atração eletrostática, precisa ser conduzida por outra técnica. Como estes trocadores iônicos apresentam caráter ácido-básico, o número de grupos de troca pode ser estimado a partir de reações ácido-base. Esta determinação está sendo conduzida, no presente momento.

O grupo ácido carboxílico, quando ionizado, é capaz de estabelecer interaçōes eletrostáticas com cátions metálicos, como os íons $\mathrm{Cd}^{+2} \mathrm{e} \mathrm{Cu}^{+2}$. Esta propriedade confere à serragem modificada características semelhantes a resinas catiônicas fracas e justifica a não adsorção, observada em pH menor que 2 (Santos et al, 2005). Kappor \& Viraraghavan (1997) estudando a adsorção de metais pesados, identificaram a presença de grupos carboxilato, em pH 5,0, em biomassa produzida a partir de Aspergillus niger. O mesmo grupo foi identificado em Datura innoxia, sendo considerado como um dos principais sítios para a adsorção dos primeiros (Drake et al, 1996).

\section{Experimentos de equilíbrio}

A Figura 3 apresenta as isotermas de adsorção dos metais $\mathrm{Cd}^{+2} \mathrm{e} \mathrm{Cu}^{+2} \mathrm{em}$ Paraju modificada. As curvas indicam o carregamento máximo para o cobre igual a $0,90 \mathrm{mmol} / \mathrm{g}$, e de $0,56 \mathrm{mmol} / \mathrm{g}$ para o cádmio. Benguella e Benaissa (2002) estudaram a adsorção de cádmio, cobre e zinco em quitina e observaram um melhor carregamento para o cobre o que foi justificado pelo fato do cobre ter menor raio iônico e, por conseguinte, poder penetrar nos menores poros, desse modo tendo um maior acesso à superfície do material. Entretanto, deve-se salientar
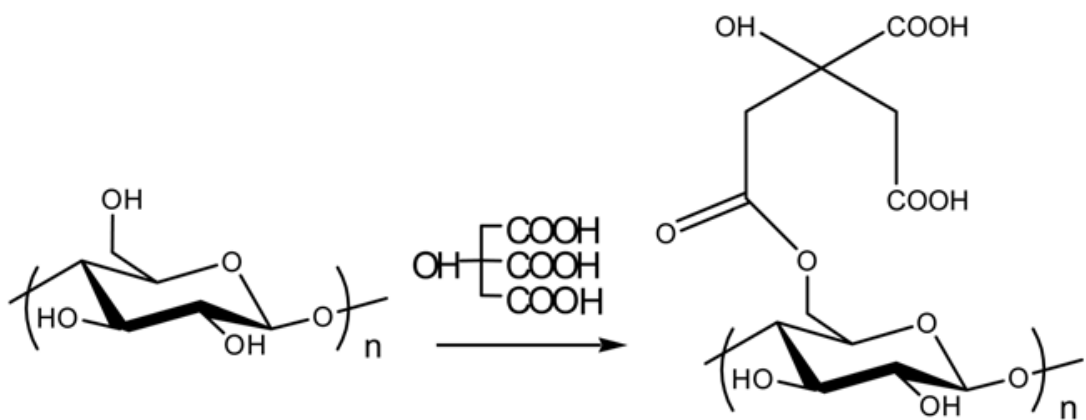

Figura 2 - Representação esquemática do(s) grupo(s) carboxilato introduzido(s) na superfície da celulose presente na serragem de Paraju 
apresentam elevada afinidade pelo íon $\mathrm{Cu}^{+2}$ formando com o este último uma série de complexos $\left(\mathrm{CuNH}_{3}{ }^{+2} ; \operatorname{logb}\right.$ $=4,0),\left(\mathrm{Cu}\left(\mathrm{NH}_{3}\right)_{2}{ }^{+2} ; \log _{2}=7,4\right)$, $\left(\mathrm{Cu}\left(\mathrm{NH}_{3}\right)_{3}{ }^{+2} ; \operatorname{logb}_{3}=10,2\right)$, $\left(\mathrm{Cu}\left(\mathrm{NH}_{3}\right)_{4}^{+2} ; \operatorname{logb}_{4}=12,3\right)$. Os valores das constantes de equilíbrio para estes complexos são pelo menos duas ordens de grandeza superiores às mesmas observadas para os complexos $\mathrm{Ni}-\mathrm{NH}_{3}$, e Cd$\mathrm{NH}_{3}$, ou seja, a maior afinidade dos grupos amino pelo íon $\mathrm{Cu}^{+2}$ também contribuem para a maior adsorção do cobre e não apenas a diferença de raios iônicos.

A adsorção dos metais foi ajustada ao modelo de Langmuir sendo calculadas a capacidade máxima de adsorção e a constante de afinidade. $\mathrm{O}$ modelo de Langmuir considera que não existe interação entre as moléculas adsorvidas, apenas entre estas e o adsorvente. Além disso, assume que a interface sólido fluido é homogênea e que a adsorção acontece em monocamadas (Hiemenz, 1986).

$\mathrm{q}_{\mathrm{eq}}=\frac{\mathrm{q}_{\text {max }} \mathrm{bC}_{\text {eq }}}{1+\mathrm{bC}_{\mathrm{eq}}}$

Onde: $\mathrm{q}_{\mathrm{eq}}$ é a quantidade de cádmio adsorvido por grama de biomassa, $\mathrm{C}_{\mathrm{eq}}$ éa concentração final de cádmio em solução, $\mathrm{q}_{\max }$ é o carregamento máximo do metal e b é uma constante relacionada com a afinidade entre o metal e a biomassa. Quanto maior o valor de b, maior a afinidade entre a biomassa e o cátion adsorvido. A Equação 3 é a forma linear dessa equação.

$\frac{\mathrm{C}_{\text {eq }}}{\mathrm{q}_{\text {eq }}}=\frac{1}{\mathrm{bq}_{\text {max }}}+\frac{\mathrm{C}_{\text {eq }}}{\mathrm{q}_{\text {max }}}$

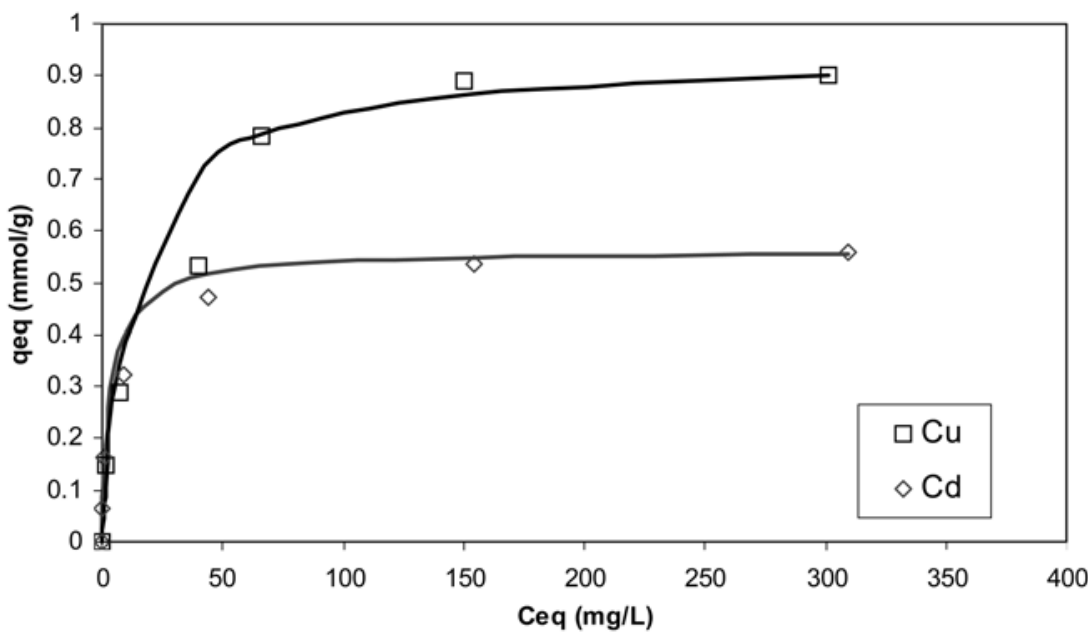

Figura 3 - Isoterma de adsorção de cádmio e cobre em Paraju tratada com ácido cítrico. Condições experimentais: 100 a $1000 \mathrm{mg} / \mathrm{L}$ do metal, $10 \mathrm{~g}$ madeira /L, pH 5,0; tamanho de partícula $<-0,074 \mathrm{~mm}$
Para a adsorção dos metais na madeira modificada, observa-se um aumento da eficiência de adsorção com o aumento do $\mathrm{pH}$. Supondo que o principal mecanismo de adsorção seja a atração eletrostática entre cátions e os grupos carboxilato ancorados na biomassa, a maior adsorção com o aumento do $\mathrm{pH}$ é devida ao maior número de grupos ácido carboxílico ionizados. Estes grupos desprotonados são os capazes de adsorver os íons metálicos. O ácido cítrico apresenta três $\mathrm{pK}_{\mathrm{a}, \mathrm{s}}$ diferentes $\left(\mathrm{pK}_{\mathrm{a}, 1}=3,13\right.$; $\left.\mathrm{pK}_{\mathrm{a}, 2}=4,76 ; \mathrm{pK}_{\mathrm{a}, 3}=6,40\right)$ (Dean, 1992) e a distribuição das diferentes espécies em função do $\mathrm{pH}$ é apresentada na Figura 5. Embora a formação de éster entre o ácido cítrico e a madeira, durante a incorporação do ácido cítrico na estrutura da biomassa, diminua o número de grupos carboxilato disponíveis para troca iônica (os grupos transformados em ésteres não são capazes de realizar troca-iônica), a distribuição das espécies em solução é um indicativo do que é possível ocorrer com o ácido ancorado na estrutura da madeira. De acordo com a Figura 5, tem-se aproximadamente $50 \% \mathrm{H}_{2} \mathrm{~L}^{-}$e $50 \% \mathrm{HL}^{-}$ ${ }^{2}$ enquanto que, em $\mathrm{pH} 4,0$, há apenas um mol de carga negativa na espécie predominante para cada mol de ácido $\left(\mathrm{H}_{2} \mathrm{~L}\right.$ ). Em outras palavras, a concentração de grupos aniônicos, capazes de efetuar a adsorção de cátions, é maior em pH 5,0 do que em $\mathrm{pH}$ 4,0. Como conseqüência, espera-se uma maior capacidade de adsorção em pH 5,0 em relação ao $\mathrm{pH} 4,0$ conforme observado experimentalmente (Rodrigues et al, 2002).

\section{CONCLUSÕES}

A biossorção de íons cádmio e cobre em madeira modificada com ácido cítrico foi avaliada. A modificação introduziu grupos funcionais no material, os quais foram caracterizados através de espectro- metria de infravermelho, onde se observou a presença de bandas características do grupo ácido carboxílico e também dos íons carboxilatos. A introdução desses grupos na biomassa com um conseqüente aumento na capacidade adsortiva do material sugere que o principal mecanismo de adsorção é a atração eletrostática entre os cátions $\mathrm{Cd}^{+2} \mathrm{e} \mathrm{Cu}^{+2}$ e os grupos carboxilato presentes na biomassa, a qual é afetada pelas condiçôes físico-químicas das soluções.

Os dados obtidos nos ensaios contendo apenas um metal mostraram um bom ajuste ao modelo de Langmuir, sen 
Tabela I - Constantes de Langmuir para as isotermas de adsorção de cádmio em biomassa. q é o carregamento máximo, b é a constante de Langmuir

\begin{tabular}{cccccc}
\hline Material & Metal & \multicolumn{2}{c}{ Constantes de Langmuir } & $\mathrm{r}^{2}$ & Referência \\
& & $\begin{array}{c}\mathrm{q}_{\max } \\
(\mathrm{mmol} / \mathrm{g})\end{array}$ & $\mathrm{b}$ & & \\
\hline $\begin{array}{c}\text { Madeira modificada com } \\
\text { ácido cítrico }\end{array}$ & $\mathrm{Cd}$ & 0,56 & 0,26 & 0,9992 & Presente trabalho \\
$\begin{array}{c}\text { Madeira modificada com } \\
\text { ácido cítrico }\end{array}$ & $\mathrm{Cu}$ & 0,94 & 0,08 & 0,993 & Presente trabalho \\
$\begin{array}{c}\text { Casca de soja modificada } \\
\text { com ácido cítrico }\end{array}$ & $\mathrm{Cu}$ & 2,44 & 0,56 & n.d. & (Marshall et al, 1999) \\
$\begin{array}{c}\text { Quitina } \\
\text { Quitina }\end{array}$ & $\mathrm{Cd}$ & 0,14 & 0,029 & n.d. & (Benguella and Benaissa, 2002) \\
$\begin{array}{c}\text { Arthrobacter sp. } \\
\text { Arthrobacter sp. }\end{array}$ & $\mathrm{Cd}$ & 0,0629 & 0,022 & n.d. & (Benguella and Benaissa, 2002) \\
\hline
\end{tabular}

n.d.: não disponível

do possível determinar sua constante de afinidade e o carregamento máximo, que foi de $0,94 \mathrm{mmol}-\mathrm{Cu} / \mathrm{g}$ e $0,56 \mathrm{mmol}-$ $\mathrm{Cd} / \mathrm{g}$. Observou-se uma diminuição na capacidade da madeira de reter cádmio na presença de cobre, sugerindo que existe uma competição entre os metais pelos grupos de adsorção.

\section{AGRADECIMENTOS}

Aos programas FINEP-RECOPE Tecnologias Limpas, RECOPE-COOPERATIVOS 2004 pelos recursos, à Fundação Gorceix e ao CNPq pelas bolsas. Agradecimento especial ao apoio da Universidade Liegè (Bélgica).

\section{REFERÊNCIAS}

BAIRD, C. Environmental Chemistry, 2 ed. New York: W.H. Freeman and Company. 557pp. 1999.

BENGUELLA, B. and BENAISSA, H. (2002). Effects of competing cations on cadmium biosorption by chitin. Colloids and Surfaces, v. 201, p. 143-150. 2002.

DEAN, J. A. Lange's handbook of Chemistry, 14 ed. New York: McGraw-Hill. 1203pp. 1992.

DRAKE, L. R. et al. Chemical modification and metal binding sudies of Datura innoxia. Environmental Science and Technology, v. 30, p. 110-114. 1996.

GABALLAH, I. et al. (1994). A new process for the decontamination of industrial efluent containing cadmium cations. In: EPD CONGRESS WARREN, G. W. (ed.) San Francisco, TMS. p. 43-52. 1994.

HIEMENZ, P. C. Principles of colloid and surface chemistry, New York: Marcel Dekker. 815 p. 1986.

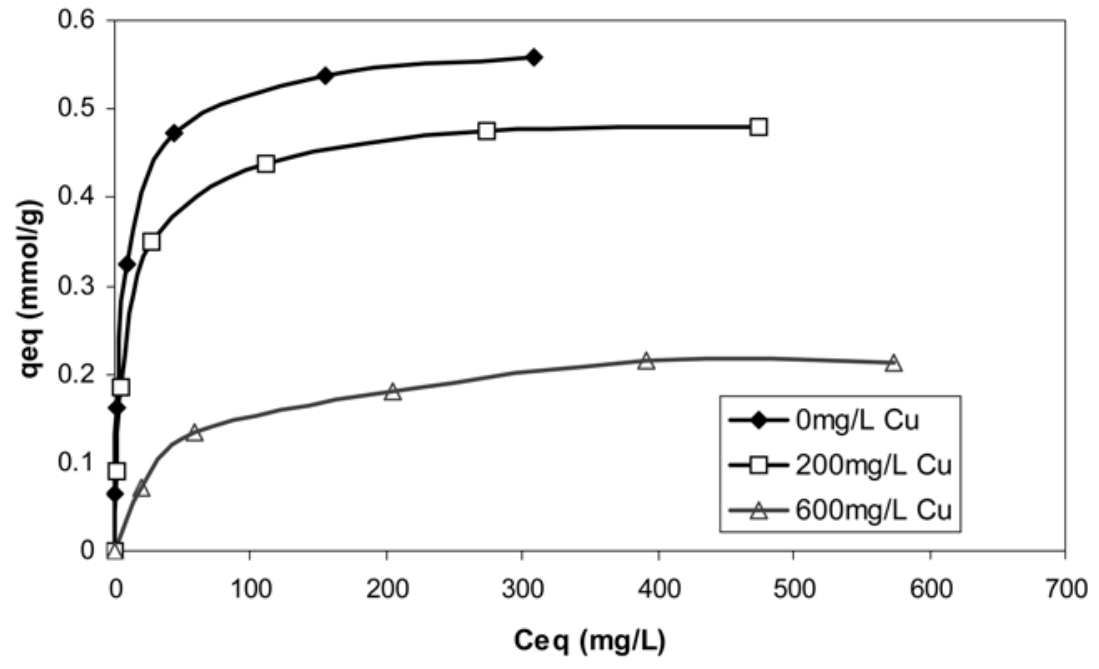

Figura 4 - Adsorção de cádmio em Paraju modificada com ácido cítrico na presença de cobre. Condições experimentais: $10 \mathrm{~g}$ madeira/L, pH 5,0; tamanho de partícula $<0,074 \mathrm{~mm}$

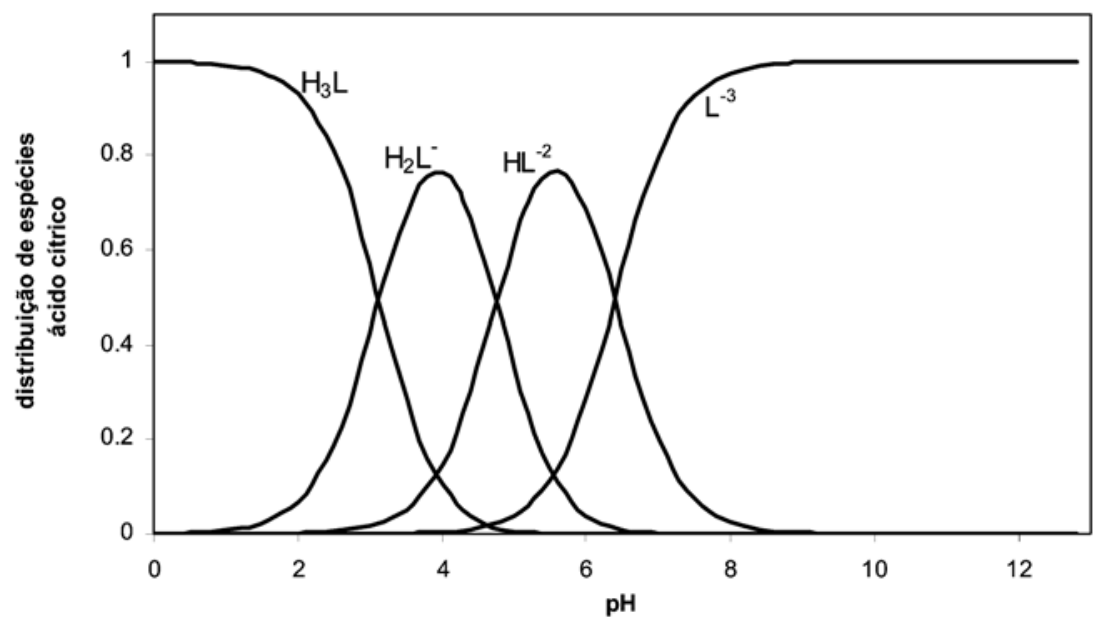

Figura 5 - Distribuição das espécies do ácido cítrico em função do pH. L indica o íon citrato (OOC) $-\mathrm{CH}_{2}-\mathrm{COH}\left(\mathrm{COO}^{-}\right)-\mathrm{CH}_{2}-(\mathrm{COO})$ 
KAPPOR, A. and VIRARAGHAVAN, T. Heavy metal biosorption sites in Aspergillus Niger. Bioresource Technology, v. 61, p. 221-227. 1997.

MARQUES, P. A. et al. Removal efficiency of $C u(I I), C d(I I)$ and $P b(I I)$ by waste brewery biomass: $p H$ and cation association effects. Desalination, v. 124, p. 137-144. 1999.

MARSHALL, W. E. et al. Enhanced metal adsorption by soybean hulls modified with citric acid. Bioresource Technology, v. 69, p. 263268. 1999.

PAGNANELLI, F. et al. Equilibrium biosorption studies in single and multi-metal systems. Process Biochemistry, v. 37, p. 115-124. 2001.

RODRIGUES, R. F. et al. Adsorção de cádmio em biomassa seca e tratada quimicamente. In:
XIX ENCONTRO NACIONAL DE TRATAMENTO DE MINÉRIOS E METALURGIA EXTRATIVA. Recipe, PE. pp. 312-317. 2002

SANTOS, L. R. G. et al. Valorization of solid wastes as sorbents for heavy metals. In: 2005 TMS ANNUAL MEETING. EPD CONGRESS. SCHLESINGER, M. E. (ed.) São Franscisco, The Minerals, Metals \& Materials Society (TMS). pp. 395-410. 2005.

VAUGHAN, T. et al. Removal of selected metal ions from aqueous solution using modified corncobs. Bioresource Technology, v. 78, p. 133-139. 2001.

YALÇINKAYA, Y. et al. Biosorption of cadmium form aquatic systems by carboxymethylcellulose and immobilized Trametes versicolor. Hydrometallurgy, v. 63 , p. $31-40.2002$.
Endereço para correspondência:

Versiane Albis Leão

Universidade Federal de Ouro Preto

Departamento de Engenharia

Metalúrgica e de Materiais

Praça Tiradentes, 20

35400-000 Ouro Preto - MG -

Brasil

Tel.: (3I) 3559-I56I

E-mail:versiane@demet.em.ufop.br

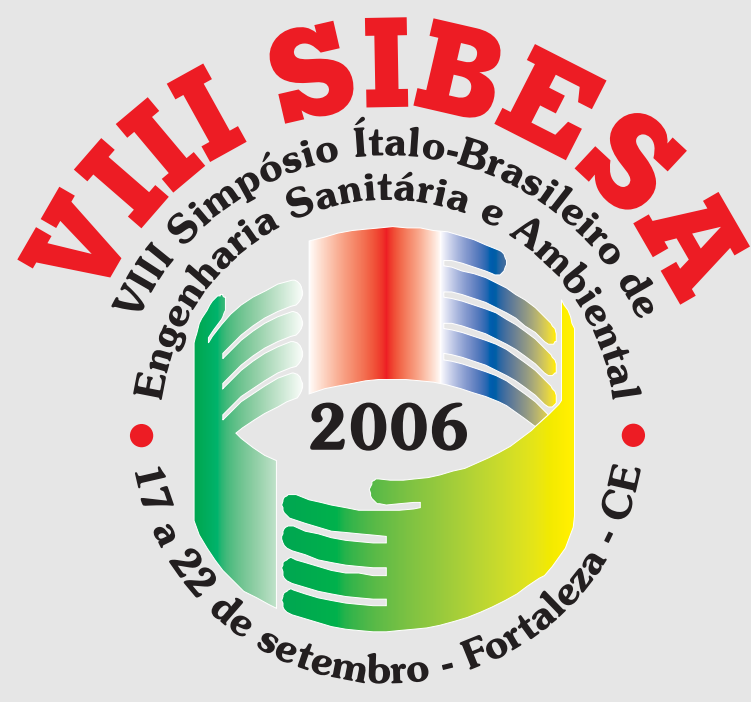

Promoção

ABES ASSOCIACĀO BRASILEIRA DE

ENGENHARIA SANITÁRIA
Capítulo Nacional da AIDIS

Realização

ABES - Seção Ceará 